\title{
EXPERIMENTAL STUDIES OF THE DISSOLUTION OF GAS BUBBLES IN WHOLE BLOOD AND PLASMA-I. STATIONARY BUBBLES $*+$
}

\author{
WEN-JEI YANG.‡ R. ECHIGO, \$ D. R. WOTTON and J. B. HWANG \\ Department of Mechanical Engineering, University of Michigan. Ann Arbor. Michigan \\ 48104. U.S.A.
}

\begin{abstract}
Gas bubbles of oxygen. carbon dioxide and nitrogen are injected into the degassed plasma and whole blood. The bubble size is measured at time intervals until the bubble is completely dissolved in the liquid. Physical quantities governing the dissolution of gas bubbles are identified and their effects are examined. The applications of the study include the dissolution of gas emboli in human body and the extracorporeal oxygenation of the blood during open heart surgery.
\end{abstract}

\section{INTRODUCTION}

GAS EMBOLISM, although relatively infrequent in medical practice, is a dreaded complication in surgical, therapeutic and diagnostic procedures when it does occur. Through an extensive survey of the literature pertinent to the problems of gas embolism, Chang and Yang (1969) have classified the causes of gas embolism into: decomposition, anesthesia, surgery, accidents, extracorporeal circulation of blood, hypothermia and fracture of bones and tissue. In other words, gas embolism arises from the existence of a gas phase which is introduced into the human body through cavitation of dissolved gas in the blood or body fluids or is forced into the human body from the environment. The most important problem related to the prevention of gas embolism is the rate at which a gas phase is re-absorbed or dissolves when it appears in the blood or body fluids. In other words, whether or not a gas phase in the human body may cause embolism depends, to a large extent, on the problem of dynamic stability and rate of growth of this gas phase appearing in the form of gas bubbles. Here is the dynamics equation of a spherical gas bubble in whole blood or plasma:

$$
R \ddot{R}+\frac{3}{2} \dot{R}^{2}=\frac{p_{g}(R)-p_{\infty}-2 \sigma / R}{\rho} .
$$

where $R$ is the bubble radius at any time $t$, $\dot{R}=\mathrm{d} R / \mathrm{d} t, \rho$ is the density of the liquid, $p_{g}(R)$ is the gas pressure inside the bubble, $p_{x}$ is the liquid at a distance from the bubble and $\sigma$ is the surface tension. It can be seen from equation (1) that the bubble is in dynamic equilibrium with the liquid if $f(R)=0$ and $\dot{R}=0$, where

$$
f(R)=p_{g}(R)-p_{\infty}-\frac{2 \sigma}{R}
$$

Whether the equilibrium of a bubble is dynamically stable or unstable is determined by the value of $\mathrm{d} f(R) / \mathrm{d} R$ for the given radius. The condition $\mathrm{d} f(R) / \mathrm{d} R<0$ indicates dynamic stability and as a result a bubble will dissolve through diffusion of gas out of the bubble. The range of equilibrium radii for which $\mathrm{d} f / \mathrm{d} R<0$ is $R_{0}<2 \sigma /\left(p_{g_{0}}-p_{x}\right)$, where $p_{g_{0}}$ is the initial pressure of the gas in a bubble of radius $R_{0}$. Yang (1970) has analyti-

* Received 16 April 1970.

tThe work was supported by a grant from the National Institute of Health.

$\ddagger$ Associate Professor of Mechanical Engineering.

§Visiting Scholar from Kyushu University, Japan. 
cally investigated the radius-time relation of a gas bubble in whole blood and plasma, and the rate of diffusion of the dissolved gas in the liquids under the condition of dynamic stability. Whereas $\mathrm{d} f(R) / \mathrm{d} R \geqslant 0$ is the condition for dynamic instability and as a result the effect of gas in a bubble may initiate the growth of the bubble. The range of equilibrium radii for which $\mathrm{d} f / \mathrm{d} R \geqslant 0$ is $R_{0} \geqslant 2 \sigma /$ $\left(p_{g_{0}}-p_{\infty}\right)$.

This paper deals with the experimental study on the dissolution of a gas bubble in degassed whole blood and plasma corresponding to the condition of $\mathrm{d} f / \mathrm{d} R<0$. Part I of this study is concerned with the physical situation in which a stationary gas bubble is situated in a quiescent plasma or blood, while part II deals with the case when there is a relative motion between the bubble and the liquid. The distinction between a gas bubble in plasma and a gas bubble in whole blood is that for the latter case chemical reaction between the dissolved gas and reduced hemoglobin takes place in the concentration boundary layer over the dissolving bubble surface.

\section{APPARATUS}

The experimental study on the dissolution of a gas bubble in degassed blood and plasma is conducted in two steps. First step is to degas the liquid. During this stage, the gas dissolved in plasma is removed and oxyhemoglobin in whole blood dissociates into reduced hemoglobin and oxygen which is removed as soon as it is formed. The occurence of dissociation of some oxyhemoglobin is evidenced by a change in the color of the blood from scarlet red to purple red. The second step involves the injection of a gas bubble into the degassed blood or plasma, followed by the measurements of the bubble size at certain time intervals until the bubble is completely dissolved in the liquid.

The equipment used in the degassing process is shown in Fig. 1. The test fluid is placed in a $250 \mathrm{ml}$ separatory funnel which has an opening on one end and a stopcock on

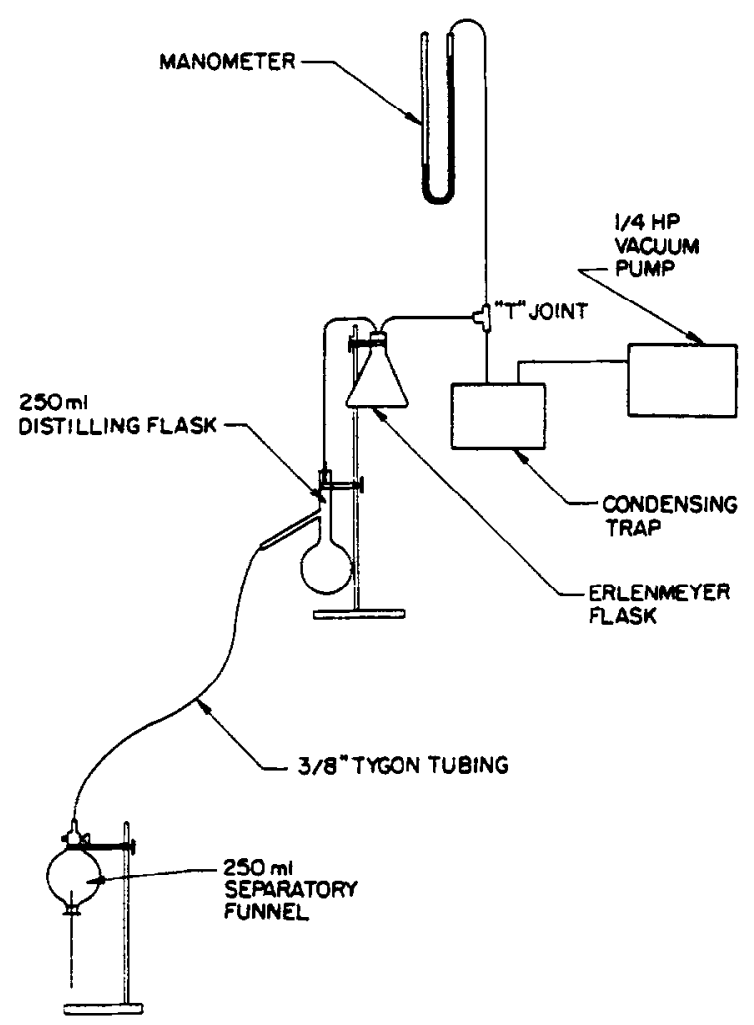

Fig. 1. Apparatus for degassing process.

the opposite end. A rubber stopper is attached to the open end. A thermometer is inserted into the fluid through a hole in the rubber stopper. During the degassing process the funnel attached to a stand is positioned with the stopcock at the top. A $1 \mathrm{~m}$ length of $\frac{3}{8}$ in. diameter tygon tubing leads from the stopcock of the funnel to a $250 \mathrm{ml}$ distilling flask, which traps fluid being carried out of the funnel during the degassing process. A $50 \mathrm{~cm}$ length of tubing leads from the top of the distilling flask to a $250 \mathrm{ml}$ Erlenmeyer flask, which retains any fluid being transported up the tubing from the distilling flask. A $50 \mathrm{~cm}$ length of tubing from the Erlenmeyer flask is connected to a ' $T$ ' joint. One branch of the ' $T$ ' leads to a mercury manometer, while the other branch is connected to a condensing trap. The condensing trap removes much of the water vapor from the air being drawn from the 
system. A $2 \mathrm{~m}$ length of $\frac{3}{8}$ in. diameter tubing leads from the condensing trap to a $\frac{3}{3}$ h.p. $\mathrm{Hy}$-Vac vacuum pump. A vacuum of approximately 28.5 in. of mercury is obtained during the degassing process.

The equipment used in injection and measurement of bubble size is illustrated in Fig. 2. The liquid in the separatory funnel serves as the medium for the diffusion of the gas bubble. The gas is drawn into a syringe with a size number 20 needle. The needle is inserted through the rubber stopper in the funnel and a small bubble is introduced into the liquid. The bubble is observed through the wall of the funnel placed on the side on a wooden support which allows the illumination of the funnel from beneath. The illumination is provided by two $15 \mathrm{~W}$ white florescent lamps. An Edscorp No. 30186 reticle is placed on the side of the funnel. The reticle has a $10 \mathrm{~mm}$ long metric scale divided into units of $0.2 \mathrm{~mm}$. A dual eyepiece Bausch and Lomb microscope is placed above the funnel and reticle. The bubble size is observed under a magnification of $10.5 \times$.

The liquid viscosity is measured using the test apparatus depicted in Fig. 3. Measuring the liquid viscosity provides a means of detec- ting any change in the physical properties of the liquid during testing. The test liquid placed in a $3.4 \mathrm{~cm}$ i.d. $14 \mathrm{~cm}$ long glass tube is allowed to flow through a capillary tube $16 \mathrm{~cm}$ long into a $100 \mathrm{ml}$ boiling flask. The time required for the liquid to fill the spherical portion of the flask is recorded. The measurements are conducted before and after each bubble test. A change in the time required to fill the flask to the chosen level indicates a proportional change in liquid viscosity. A substantial change in liquid viscosity may indicate the deterioration of the biological fluid and thus will influence the rate of dissolution of the gas bubble.

\section{EXPERIMENTAL PROCEDURE}

The experiment begins with the degassing of the test liquid. Two stands are used. one to hold the separatory funnel and the other to hold the distilling flask and the Erlenmeyer flask, as illustrated in Fig. 1. The base of the second stand is placed about $76 \mathrm{~cm}$ above that of the first stand. The level of the Erlenmeyer flask is approximately $35 \mathrm{~cm}$ above that of the distilling flask. The positioning of the containers at different levels allows much of the liquid transported into the tubing to flow back into

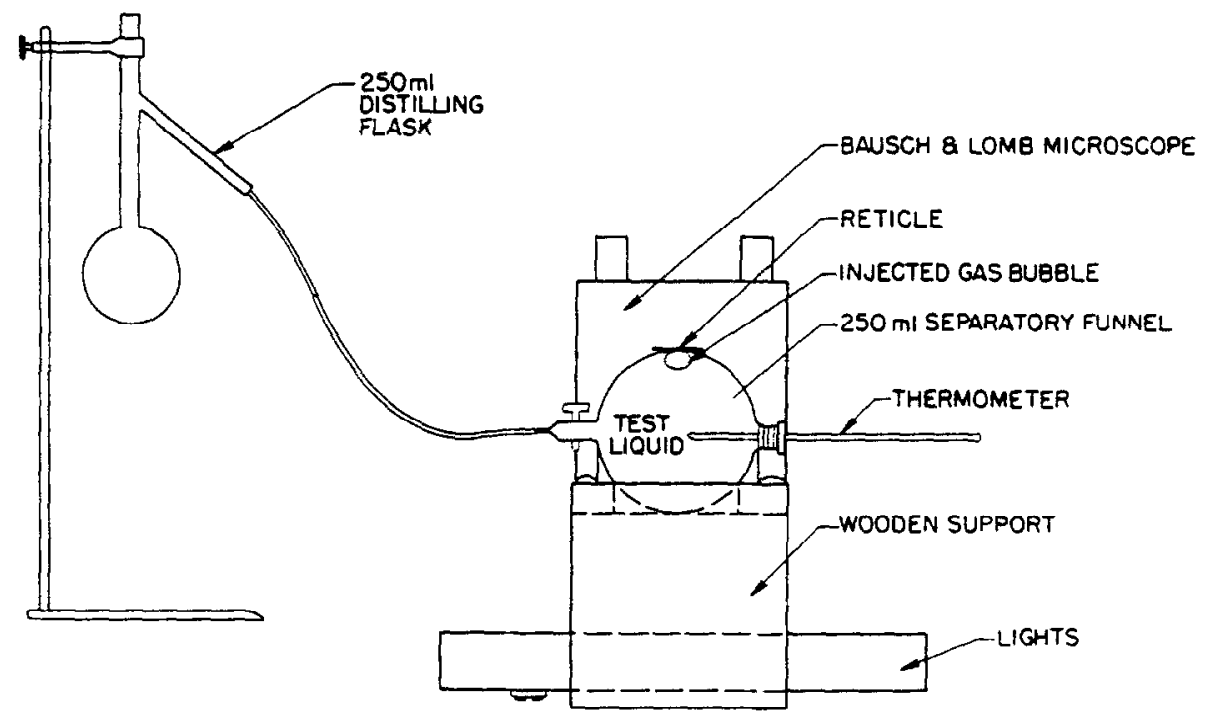

Fig. 2. Bubble measurement set up. 


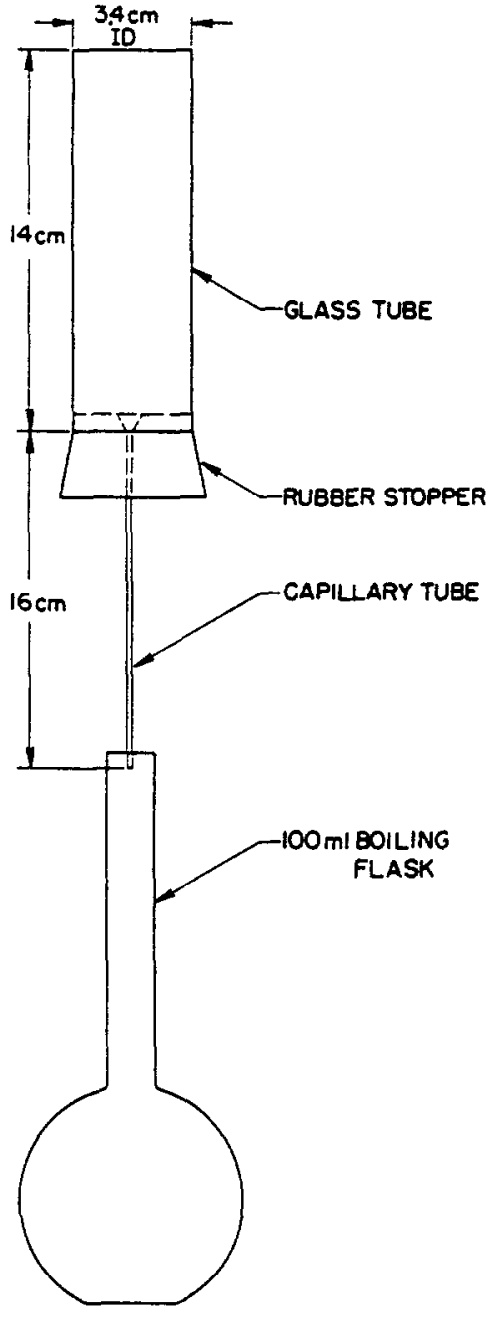

Fig. 3. Apparatus for viscosity measurement.

its original container. $200 \mathrm{ml}$ of liquid is placed in the separatory funnel and in the distilling flask. The vacuum pump is turned on until the bubbles formed during the pumping process fill the containers and the tubing above the containers. Then, the pump is turned off until the liquid transported by the rapid bubbling in the containers has settled back into the original container. The on-andoff operation of the pump is repeated until the bubbling rate becomes low enough that the transportation of the liquid from the separatory funnel has ceased. The pump is then left running to complete the degassing process. After a bubble has been injected and absorbed the pumping is resumed prior to another bubble injection. For plasma, the pumping times for the first run and subsequent runs are $12 \mathrm{hr}$ and $1 \mathrm{hr}$, respectively. In case of dog blood, these pumping times are $10 \mathrm{hr}$ and $1 \mathrm{hr}$, respectively. It was observed that bubbling processes diminished in approximately $8 \mathrm{hr}$. The vacuum pressure is read off the mercury manometer.

When the degassing is complete, the vacuum pump is turned off. Liquid in the distilling flask is transferred back to the separatory funnel through the tubing connecting the two containers. The distilling flask is inclined and the liquid is allowed to run down the connecting tube. The pouring is continued until the liquid has filled half the length of the tubing leading to the separatory funnel. Then, the tubing leading from the condensing trap to the vacuum pump is disconnected at the condensing trap. The pressure in the flasks returns to the ambient pressure. The rubber stopper is removed from the distilling flask which is now moved to the top of the second stand. The separatory funnel is removed from the first stand and placed on the wooden support as shown in Fig. 2. A gas bubble is then injected through the rubber stopper into the degassed liquid in the separatory funnel. The bubble diameter is measured at frequent intervals. The equipment is returned to the arrangement depicted in Fig. 1 after the gas bubble is completely dissolved in the liquid. The level of the liquid in both the separatory funnel and distilling flask are adjusted to contain $200 \mathrm{ml}$ of the liquid before next run is started.

The viscosity of the liquid is measured periodically to check any possible change in the liquid property resulting from the degassing process or its continuous exposure to the room temperature and pressure.

\section{RESULTS AND DISCUSSION}

Since the bubbles are in contact with the glass container, they cannot be called spheri- 
cal bubbles in the strict sense of the word. Rather they tend to assume a shape similar to an oblate spheroid. Due to the application of liquid pressure, the dimension of the bubble is reduced in the direction normal to the concontainer surface and increased along the container surface. Thus the measured bubble size was reduced to an equivalent size of a spherical bubble and plotted in radius-time curves.

In the experiments, the liquids including the blood and plasma were degassed under 28.80 to 28.95 in. $\mathrm{Hg}$ negative pressure. During the degassing process the liquid temperature fell below the room temperature by 2 to $3^{\circ} \mathrm{F}$ due to a decrease in the vapor pressure above the liquid. As a result, the liquid temperature gradually restored to the level of the room temperature during the observation of dissolution of a gas bubble.

Oxygen is carried by the blood mainly in reversible chemical combination, rather than in physical solution. After human and dog bloods are degassed the color turns purple, indicating that some oxyhemoglobin is dissociated to the reduced form or hemoglobin. It is estimated from the vacuum pressure and the oxyhemoglobin dissociation curves of human blood (Fenn and Rahn, 1964) that after degassing pressure the concentration of oxygen in association with hemoglobin has been reduced to few (approximately 2) per cent of its saturated concentration under one atmosphere. While an oxygen bubble was injected into partially degassed blood, the color of the blood in the vicinity of the bubble very quickly changed from purple to scarlet red. This is the evidence that oxygen contained in the bubble has diffused into the blood and formed a thin concentration boundare layer over the bubble. In the boundary layer hemoglobin of the red cells combined with the dissolved oxygen in the form of the scarlet red compound, oxyhemoglobin. It was observed that during this period the bubble shrunk very rapidly (too rapidly to read its size). However, following the formation of a scarlet red ring around the bubble, the bubble disolved in the blood rather slowly, indicating that reversible chemical combination in the concentration boundary layer is nearly in equilibrium, and that the dissolution of the oxygen bubble is mainly due to diffusion through the boundary layer.

Figures 4 and 5 show the radius-time curves of oxygen bubbles in human and dog bloods, respectively. Because of very rapid shrinkage of an oxygen bubble immediately following injection into the bloods, and also a difficulty in reading bubble size due to the color of the

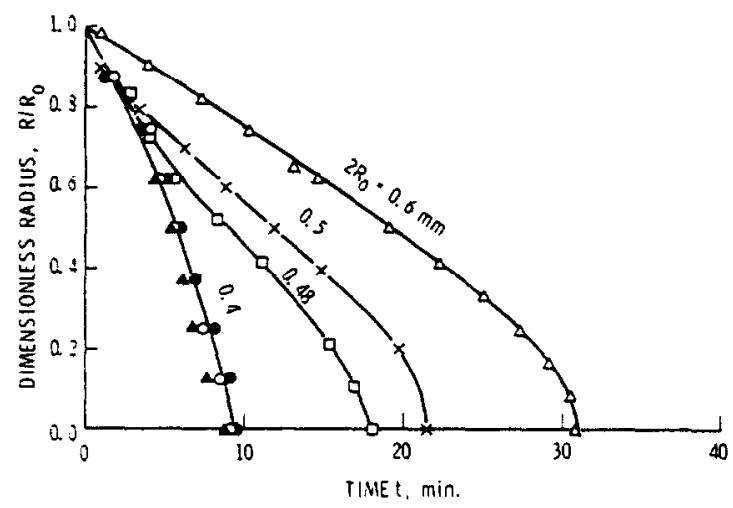

Fig. 4. Radius-time relation of oxygen bubbles in degassed human blood.

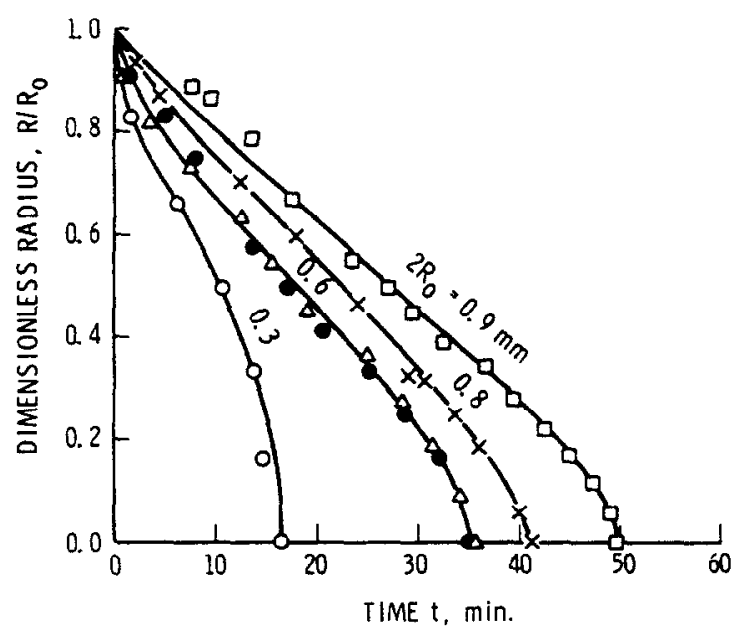

Fig. 5. Radius-time relation of oxygen bubbles in degassed dog blood. 


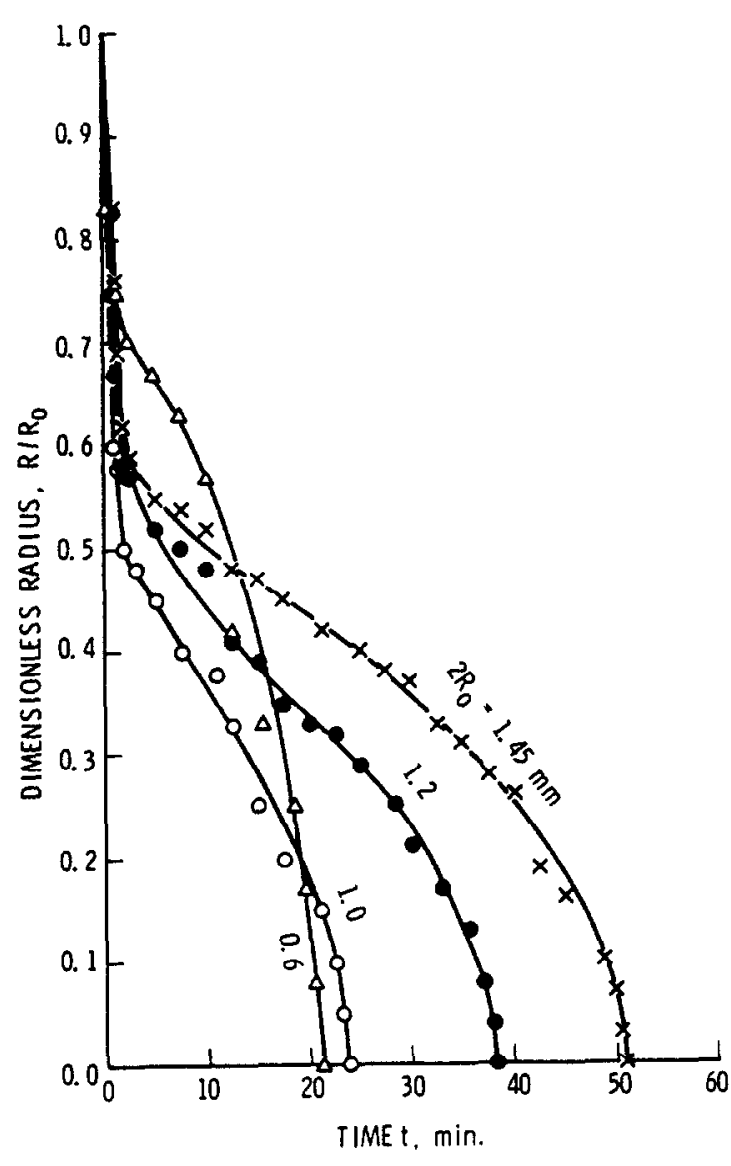

Fig. 6. Radius-time relation of carbon dioxide bubbles in degassed plasma. bloods, the initial bubble size could not be obtained. The first readings were taken between 0.5 and $3 \mathrm{~min}$ after injection. The initial radius $R_{0}$ in Figs. 4 and 5 was obtained by extrapolation of the data to zero time. Through the extrapolation, very rapid shrinkage of oxygen bubbles in the very short time following injection is not seen in the figures. However, the general appearance of the radius-time curves should be analogous to those of carbon dioxide bubbles in plasma, to be shown later in Fig. 6. It is seen in Figs. 4 and 5 that the times for complete dissolution of oxygen bubbles of the same initial size in human and dog bloods are about the same.

Like oxygen, carbon dioxide is carried by the blood mainly in reversible chemical combination. The dissolution of carbon dioxide bubbles was conducted in plasma for two reasons. One is for convenience in visual observation and the other is to include only the effects of chemical reactions in the plasma. In case of carbon dioxide in the blood, several types of chemical reaction would occur in the red cells and in the plasma (Fenn and Rahn, 1964).

When a carbon dioxide bubble is injected in the degassed plasma, a concentration boundary layer of the dissolved carbon dioxide is formed around the bubble. Most of the carbon

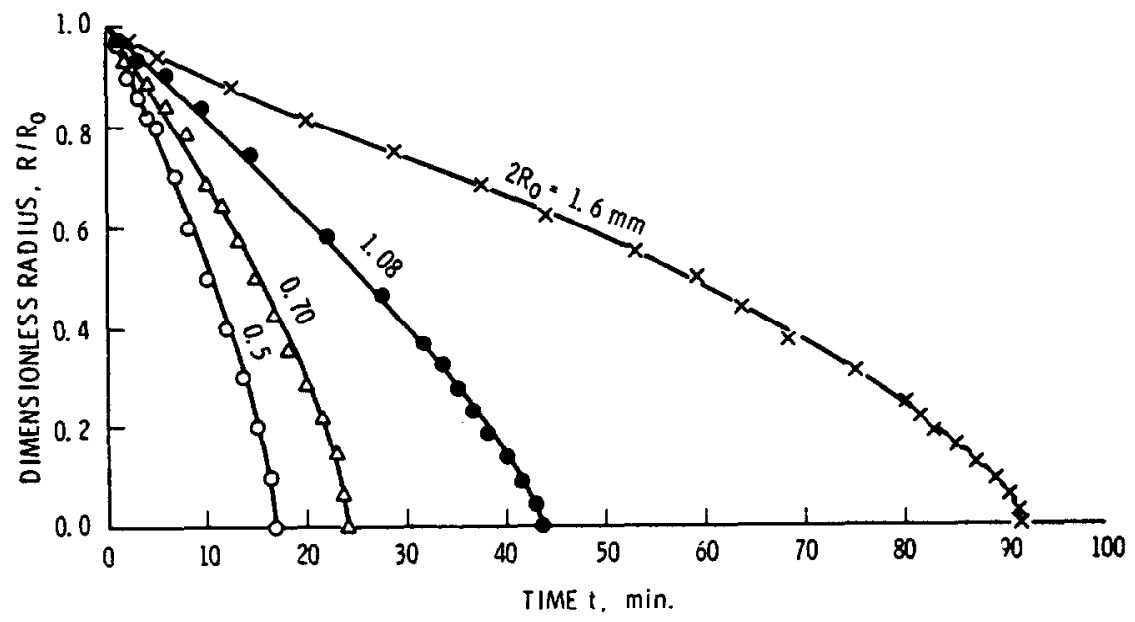

Fig. 7. Radius-time relation of oxygen bubbles in degassed plasma. 
dioxide quickly combines with water to form carbonic acid. This is a loose, reversible combination due to the reversible formation of bicarbonate ions. The rapid dissolution of carbon dioxide bubbles shown in Fig. 6 indicate the occurance of chemical reaction immediately following injection. The subsequent shrinkage of the bubbles until complete dissolution is due to diffusion mechanism. The shorter lifetime of carbon dioxide bubbles in the plasma than that of oxygen bubbles in the bloods, suggests higher diffusivity of carbon dioxide in the plasma than oxygen in the bloods.

The dissolution of oxygen and nitrogen gases in the plasma are due to diffusion alone. The radius-time curves are illustrated in Figs. 7 and 8. A comparison of the figures indicates that nitrogen gas bubbles have the longest lifetime in the plasma, and consequently also in the bloods, than oxygen and carbon dioxide. To be sure, the speed of dissolution depends on the magnitude of diffusion coefficient rather than solubility.

One observation is common in Figs. 4 through 8 . That is, the rate of shrinkage of bubbles is accelerated near the completion of dissolution, as evidenced by the steeper slope of the radius-time curves.

Figure 9 presents the absolute viscosities of the plasma and dog blood measured by the

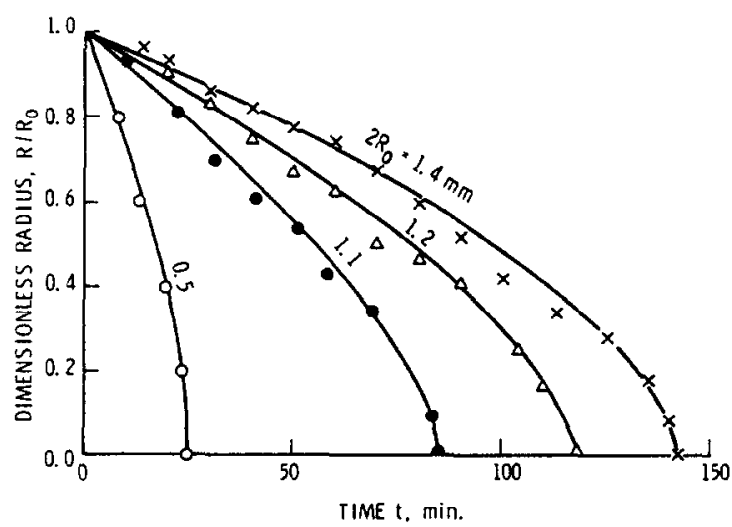

Fig. 8. Radius-time relation of nitrogen bubbles in degassed plasma.

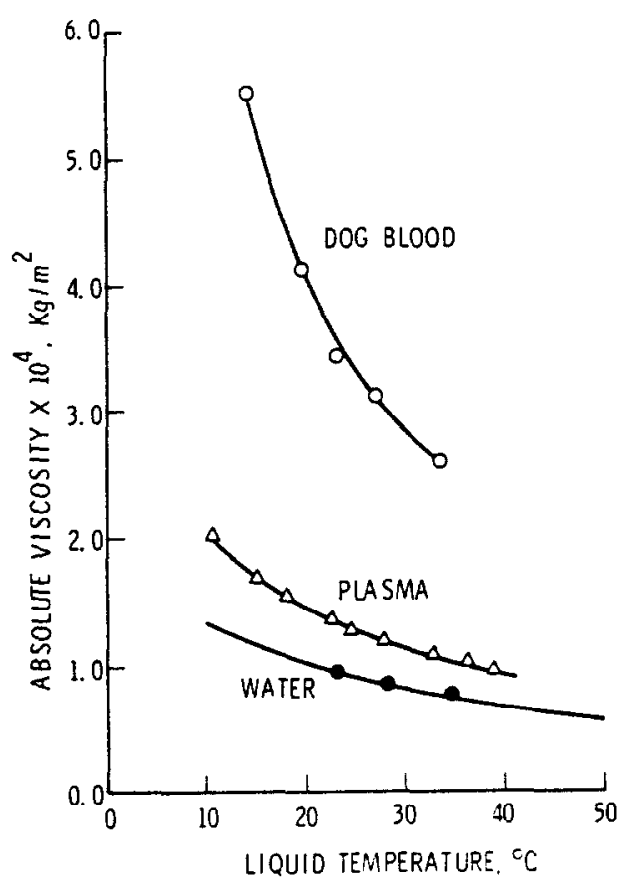

Fig. 9. Viscosity of liquids measured under one atmospheric pressure.

apparatus shown in Fig. 3. The confidence in the viscosity test apparatus and technique was borne out by comparing the measured viscosity of water (shown by dots) and the data given in handbooks (shown by solid line). No appreciable deviations in the liquid viscosity from that shown in Fig. 9 were observed before and after the degassing process and bubble dissolution test.

Acknowledgement - The investigation is supported by a grant (Grant No. 1 ROI HE 12708) from the National Institute of Health for which the authors are grateful.

\section{REFERENCES}

Bird, R. B.. Stewart. W. E. and Lightfoot. En. N. (1960) Transport Phenomena. Part 3, Wiley. New York.

Chang. K. S. and Yang. W. J. (1969) Survey of literature related to the problems of gas embolism in human body. J. Biomechanics 2, 299-312.

Fenn, W. O. and Rahn, H. (Editors) (1964) Handbook of Physiology, Respiration. Vol. 1, p. 777. American Physiological Society. Ithica, New York.

Yang. W.-J. (1971) Dynamics of gas bubbles in whole blood and plasma. J. Biomechanics 4. 119. 